\title{
ON RANDOM SOLUTIONS OF FREDHOLM INTEGRAL EQUATIONS
}

\author{
BY A. T. BHARUCHA-REID ${ }^{1}$ \\ Communicated by Richard Bellman, October 27, 1959
}

1. Introduction. In this note we consider the solution of the random operator equation

$$
(T(\omega)-\lambda I) x(u, \omega)=y(u, \omega)
$$

when the operator $T(\omega)$ is a random integral operator of the Fredholm type on a concrete Banach space of generalized random variables. In [3] (the proofs will appear in [4]) we announced some results in the theory of random operator equations; and we refer to the above paper, or to [6] for all definitions. We begin our study of concrete random operator equations with the Fedholm integral equations for the following reasons: (1) there is a well-developed theory of these equations (cf. [11] and [13]), (2) the relationship between these equations and Volterra integral equations, algebraic systems of linear equations, and Sturm-Liouville systems of differential equations, and (3) the widespread occurrence of these equations in mathematical physics and other branches of applied mathematics.

2. The stochastic boundary value problem for integral equations. In general, the classical (i.e., deterministic or nonstochastic) boundary value problem can be described as follows: Given a functional equation

$$
\mathcal{P}(x(u))=0
$$

defined on a domain $U$, with boundary $F$, in $k$-dimensional Euclidean, space $R_{k}$, find a function $x(u)$ satisfying Equation (2) in $U$ and taking prescribed values on the boundary $F$; that is $\lim _{u \rightarrow u_{0} \in F} x(u)=\gamma\left(u_{0}\right)$. The functional $P$ in (2) is a mapping of the abstract space of function $x(u)$ onto itself.

However, in many boundary value problems arising in various field of applications, the boundary conditions cannot be expressed by a single well-determined or known function $\gamma\left(u_{0}\right)$; hence it is necessary to consider this problem within the framework of probability theory, and consider a collection or set of functions, say $\Gamma=\{\gamma(u, \omega), \omega \in \Omega\}$,

1 This research was supported in part by funds provided under Contract No. DA-04-200-ORD-651 with the Office of Ordnance Research, U. S. Army. 
where $\omega$ is an element in some probability space $(\Omega, a, \mu)$. Therefore, in stochastic boundary value problems an event consists of the choice ${ }_{j}$ of an element $\omega_{0} \in \Omega$, with probability $\mu(B)=\operatorname{Pr}\left\{\omega_{0} \in B\right\}, B \in Q$, which in turn specifies the boundary condition $\gamma\left(u, \omega_{0}\right) \in \Gamma$ that is to be imposed.

In connection with stochastic boundary value problems for partial differential equations, Kampé de Fériet [8] has given a definition of a random solution of Equation (2). In order to establish a connection between random solutions of Equation (2) (or Equation (1)) and generalized random variables, we introduce the following definition. Let $U$ be a bounded closed subset in Euclidean space $R_{k}$ with Lebesgue measure $m,(\Omega, Q, \mu)$ a probability space, and $(\mathfrak{X}, \mathfrak{C})$ a measurable space, where $\mathfrak{X}$ is a Banach space of generalized random variables, and $\mathcal{C}$ is a $\sigma$-algebra of all Borel subsets of $\mathfrak{X}$.

Definition. The mapping $x(u, \omega)$ of the product space $U \times \Omega$ into the Banach space $\mathfrak{X}$ is a random solution of Equation (2) (or Equation (1)) corresponding to the random boundary condition $\gamma(\omega) \in \Gamma$ if

(i) $x(u, \omega)$ is a generalized random variable with values in $\mathfrak{X}$;

(ii) for each arbitrary, but fixed, $\omega_{0} \in \Omega-\Lambda$ where $\mu(\Lambda)=0$, the mapping (or sample function) $x\left(u, \omega_{0}\right) \in \mathfrak{X}$ is a solution of Equation (2) (of Equation (1)) in $U$ taking the value $\gamma\left(\omega_{0}\right)$ on the boundary $F$; then we have (cf. [13, p. 19, Theorem 5]);

(iii) $x(u, \omega)$ is $\mu$-measurable.

In view of the above definition, a random solution is a $\mu$-measurable generalized random variable (or random element) with values in the Banach space $\mathfrak{X}=\mathfrak{X}(U, m)$; hence the theory of generalized random variables developed by $O$. Hanš [1], E. Mourier [10], and others, can be used to investigate the solutions of random boundary value problems in Banach spaces.

In the case of integral equations, as here considered, the choice of an element $\omega \in \Omega$ specifies a fundamental domain $D_{\omega} \subseteq U$ over which the integral is defined. Hence the mapping $\gamma(\omega)$ is a mapping of the space of elementary events $\Omega$ into the set $\Gamma$ of all possible domains in $U$ over which the integral can be defined. Hence, the fundamental domain $D_{\omega}$ is a random set; or in the case of rectangular sets, the end points of the interval are ordinary random variables.

3. Random Fredholm integral equations. Let $U$ be a bounded or unbounded interval in $R_{k}$ (hence we can have $U \equiv R_{k}$ ), and let $m$ be Lebesgue measure on $U$. As before, let $(\Omega, a, \mu)$ be a probability space; and let $\left(L_{\Phi}, \mathcal{C}\right)$ be a measurable space, where $L_{\Phi}$ is the Orlicz space $L_{\Phi}(U, m)$. We shall work in Orlicz spaces since much of the 
recent work in the theory of integral equations (cf. [9] and [13]) has been carried out in these spaces.

The stochastic version of the nonhomogeneous Fredholm integral equation of the second kind, i.e.

$$
\int K(l, u) x(u) d m(u)-\lambda x(t)=y(t)
$$

when random boundary conditions are imposed, can be written as

$$
\int_{D_{\omega}} K(t, u) x(u, \omega) d m(u)-\lambda x(t, \omega)=y(t, \omega) .
$$

In Equation (4) the unknown function $x(t, \omega)$ and the known function $y(t, \omega)$ are generalized random variables with values in the separable Orlicz space ${ }^{2} L_{\Phi}$. (We refer to $[1 ; 2]$ or $[4]$ for the definition of random variables with values in an Orlicz space.) We shall assume that the known function $y(t, \omega)=y(t)$ for all $\omega \in \Omega$, and that the kernel function $K(t, u)$, which is deterministic (i.e., it does not depend on $\omega)$, is an $m \times m$-measurable function on $D_{\omega} \times D_{\omega}$. The parameter $\lambda$ is an arbitrary complex number.

We shall also consider the adjoint random Fredholm integral equation

$$
\int_{D_{\omega}} K(u, t) x^{*}(u, \omega) d m(u)-\lambda x^{*}(t, \omega)=y^{*}(t)
$$

in the adjoint space $L_{\Phi}^{*}\left(\equiv L_{\Psi}\right)$.

In operator form Equation (4) becomes $(T(\omega)-\lambda I) x=y$, where

$$
T(\omega) x=\int_{D_{\omega}} K(t, u) x(u, \omega) d m(u)
$$

is a random integral operator on the Orlicz space $L_{\Phi}\left(D_{\omega}, m\left(D_{\omega}\right)\right)$. We assume that the integral exists in the sense of Lebesgue for almost every $\omega \in \Omega$.

We first consider some properties of the random integral operator $T(\omega)$. Let us rewrite (6) in the form

$$
\int_{U} K(t, u) h(u, \omega) d m(u)
$$

where

2 The Orlicz space under consideration will always be separable since the measure $m$ is Lebesgue measure. 


$$
\begin{aligned}
h(u, \omega) & =x(u, \omega), & & \text { for } u \in D_{\omega}, \\
& =0, & & \text { for } u \in U-D_{\omega} .
\end{aligned}
$$

The function $h(u, \omega)$ can also be written as $h(u, \omega)=\chi_{D_{\omega}}(u) x(u, \omega)$, where $\chi_{D_{\omega}}(u)$ is the characteristic function of the random set $D_{\omega}$. Since $K(t, u)$ is a continuous function of $t, u \in D_{\omega}$, and is, by hypothesis, an $m\left(D_{\omega}\right) \times m\left(D_{\omega}\right)$-measurable function on $D_{\omega} \times D_{\omega}$, and if we restrict our attention to measurable solutions $x(u, \omega)$, then it is clear that the following proposition is true:

$A$ sufficient condition for $h(u, \omega)$, and consequently $T(\omega)$, to be measurable is that the characteristic function $\chi_{D_{\omega}}(u)$ be measurable, ${ }^{3}$ and also the random solution $x(u, \omega)$.

We introduce the following:

Definition (cf. [13]). The kernel $K(t, u)$, measurable on $D_{\omega} \times D_{\omega}$, has the property $(\mathrm{P})$ relative to the Orlicz space $L_{\Phi}\left(D_{\omega}, m\left(D_{\omega}\right)\right)$ when

$$
r(t)=\int_{D_{\omega}}|K(t, u) x(u, \omega)| d m(u) \in L_{\Phi}\left(D_{\omega}, m\left(D_{\omega}\right)\right) .
$$

As before, the integral is assumed to exist in the sense of Lebesgue for almost every $\omega \in \Omega$. We have

TheOREM 1. If the kernel $K(t, u)$ has the property $(\mathrm{P})$, then the random integral operator $T(\omega)$ defined by (7) is a linear random operator on $L_{\Phi}$ into $L_{\Phi}$.

THEOREM 2. If the kernel $K(t, u)$ has the property $(\mathrm{P})$, the linear random operator $T(\omega)$ defined by (7) is a bounded random operator on $L_{\Phi}$ into $L_{\Phi}$.

Since the kernel $K(t, u)$ has the property (P), Theorem 1 follows for every $\omega \in \Omega$ from the classical result $[13$, p. 227]; and Theorem 2 follows from the classical result $[13$, p. 228] applied to every $\omega \in \Omega$ separately.

The above results can be summarized as follows: the random (Fredholm) integral operator (6) on the Orlicz space $L_{\Phi}$ will be a measurable random endomorphism if (i) the kernel $K(t, u)$ has the property $(\mathrm{P})$, and (ii) the random solution $x(u, \omega)$ and the characteristic function of the random set $D_{\omega}$ are measurable with respect to the same $\sigma$-algebra of Borel subsets of $\Omega$.

If we assume that $T(\omega)$ is compact an application of the classical theory yields

${ }^{3} \chi_{D_{\omega}}(u)$ will be measurable if the random set $D_{\omega}$ is measurable. 
TheOREM 3. For every fixed $\omega \in \Omega$ the number of eigenvalues of the nonhomogeneous random Fredholm integral equation is zero, finite, or denumerably infinite; and in the latter case $\lim _{n \rightarrow \infty} \lambda_{n}=0$.

This result also holds for the adjoint random integral equation, since it depends on the compactness of $T(\omega)$, and $T^{*}(\omega)$ is compact if $T(\omega)$ is compact.

In [4] we established the existence of a $\mu_{0}$-measurable random resolvent operator $R_{\lambda}(T, \omega)=(T(\omega)-\lambda I)^{-1}$, where $\mu_{0}$ is the measure of the set $\Omega_{0}(\lambda)=\{\omega:|\lambda|>\|T(\omega)\|\}$. Using this result and the corresponding result for the operator $R_{\lambda}\left(T^{*}, \omega\right)$, together with classical theory, we have

THEOREM 4. For every $\omega \in \Omega_{0}$ every complex number $\lambda \neq 0$ belongs either to the resolvent set of $T(\omega)$ and $T^{*}(\omega)$, or it is an eigenvalue of $T(\omega)$ and $T^{*}(\omega)$. In the first case the random Fredholm integral equations

$$
\begin{gathered}
\int_{D_{\omega}} K(t, u) x(u, \omega) d m(u)-\lambda x(t, \omega)=y(t), \\
\int_{D_{\omega}} K(u, t) x^{*}(u, \omega) d m(u)-\lambda x^{*}(t, \omega)=y^{*}(t)
\end{gathered}
$$

have for every $y(t) \in L_{\Phi}$ and $y^{*}(t) \in L_{\Phi}^{*} \equiv L_{\Psi}$ uniquely determined solutions

$$
\begin{gathered}
x(u, \omega)=R_{\lambda}(T, \omega) y(t) \in L_{\Phi}, \\
x^{*}(u, \omega)=R_{\lambda}\left(T^{*}, \omega\right) y^{*}(t) \in L_{\Psi} .
\end{gathered}
$$

4. Random solutions by successive approximations. The theory of random contraction transformations as developed by O. Hanš [5; 7] and A. Špaček [12] can be utilized to prove

THEOREM 5. Consider the random Fredholm integral equation (4) in the Orlicz space $L_{\Phi}\left(D_{\omega}, m\left(D_{\omega}\right)\right)$, where the kernel $K(t, u)$ is measurable on $D_{\omega} \times D_{\omega}$, with $|K(t, u)|<M$, and for all $\omega \in \Omega y(t) \in L_{\Phi}$ is known. If $|\lambda|>m\left(D_{\omega}\right) M$, where $m\left(D_{\omega}\right)$ is the Lebesgue measure of $D_{\omega}$, then for every fixed $u \in D_{\omega}$ there exists a mapping $\tau_{\lambda}(\omega)$ from $\Omega$ into $\mathfrak{X}$ which is a generalized random variable, and the solution of Equation (4).

We remark that the solution can be found by successive approximations, the $n$th approximation being given by

$$
x_{n}(t, \omega)=\frac{1}{\lambda}\left\{\int_{D_{\omega}} K(t, u) x_{n-1}(u, \omega) d m(u)-y(t)\right\},
$$


where $x_{0}(u, \omega)$ is an arbitrary generalized raneom variable with values in $L_{\Phi}$.

\section{REFERENCES}

1. A. T. Bharucha-Reid, On random elements in Orlicz spaces, Bull. Acad. Polon. Sci. Cl. III vol. 4 (1956) pp. 655-657.

2. ——Über die Konvergenz der Folgen von verallgemeinerten zufälligen Grössen in Orliczschen Räumen, Bull. Acad. Polon. Sci. Ser. des Sci. Math. Astr. et Phys. vol. 7 (1959) pp. 425-427.

3. - On random operator equations in Banach spaces, Bull. Acad. Polon. Sci. Ser. des Sci. Math. Astro. et Phys. vol. 7 (1959) pp. 561-564.

4. - On random solutions of integral equations in Banach spaces, Trans. Second Prague Conference, 1959, 1960, in press.

5. O. Hanš, Reduzierende zufällige Transformationen, Czechoslovak Math. J. vol. 7 (1957) pp. 154-158.

6. - Generalized random variables, Trans. First Prague Conference, 1956, 1957, pp. 105-125.

7. - Random fixed point-theorems, Trans. First Prague Conference, 1956, 1957, pp. 105-125.

8. J. Kampé de Fériet, Random solutions of partial differential equations, Proceedings of the Third Berkeley Symposium, vol. 3, 1956, pp. 199-208.

9. M. A. Krasnosel'skiY and Ya. B. RutitskiY, Convex functions and Orlicz spaces (in Russian), Moscow, 1958.

10. E. Mourier, Elements aléatoires dans un espace de Banach, Ann. Inst. H. Poincaré vol. 13 (1953) pp. 161-244.

11. F. Riesz and B. Sz. Nagy, Leçons d'analyse fonctionelle, Budapest, 1953.

12. A. Špaček, Zufällige Gleichungen, Czechoslovak Math J. vol. 5 (1955) pp. 462-466.

13. A. C. Zaanen, Linear analysis, New York, 1953.

UNIVERSITY OF OREGON 